\title{
La evaluación institucional en el contexto de la universidad ecuatoriana
}

\section{Institutional evaluation in the context of the Ecuadorian university}

Fabián Eduardo Terán-Cano

https://orcid.org/0000-0002-9909-7345

Karina Elizabeth Tituaña-Dávila

https://orcid.org/0000-0002-4630-3651

Universidad Ibeoamericana del Ecuador, Ecuador

Autor por correspondencia: fabianteran@hotmail.com; kary_tn@ hotmail.com

Fecha de recepción: 27 de octubre de 2019 - Fecha de aceptación: 27 de febrero de 2020

\section{Resumen}

La evaluación institucional está ligada directamente a la toma de decisiones, con el objetivo de lograr la mejora continua de las instituciones universitarias, sus programas, resultados de aprendizaje, el desempeño de los profesores, y con especial énfasis en el entorno del aprendizaje, que permita tener un conocimiento de la realidad que atraviesa la institución, y, al mismo tiempo generar una cultura de evaluación en el ámbito universitario. Evaluar implica necesariamente establecer comparaciones a través de analizar las diferentes posturas presentes en los modelos de evaluación institucional propuestos por autores y organizaciones como Scriven, Stake, Sttufflebeam, EFQM, CINDA, CEAACES, CACES. En este artículo de investigación se realiza el abordaje desde la perspectiva teórica, analizando el estado del arte de los modelos de evaluación institucional, develando su significado para determinar coincidencias y divergencias especialmente en aquellos aplicados en la universidad ecuatoriana, que no han alcanzado el propósito de la calidad educativa, lo que obliga a repensar los enfoques y dimensiones que se consideran para contribuir de una manera asertiva al mejoramiento de los procesos de enseñanza y demás funciones sustantivas de la universidad.

Palabras Clave: modelos; evaluación institucional; universidad; calidad; aseguramiento

\begin{abstract}
Institutional evaluation is directly linked to decision-making, with the aim of achieving continuous improvement of university institutions, their programs, learning outcomes, teacher performance, and with special emphasis on the learning environment, which allows have a knowledge of the reality that the institution is going through and at the same time generate a culture of evaluation at the university level. Evaluating necessarily implies establishing comparisons through analyzing the different positions present in the institutional evaluation models proposed by authors and organizations such as Scriven, Stake, Sttufflebeam, EFQM, CINDA, CEAACES, CACES. This research article is approached from a theoretical perspective, analyzing state of the art models of institutional evaluation, revealing their meaning which allows the determining of the similarities and divergences especially in those models applied in the ecuadorian university that have not reached the goal of educational quality, which forces us to rethink approaches and dimensions to efectively contribute to the improvement of the teaching processes and other main roles of the university
\end{abstract}

Key words: models; institutional evaluation; university; quality; assurance 


\section{Introducción}

La evaluación y acreditación de las universidades y escuelas politécnicas inicia formalmente en el Ecuador a inicios del siglo XXI, pero este proceso ya fue aplicado hace varios años en Estados Unidos de Norteamérica, Inglaterra, Francia, España, con resultados positivos para el mejoramiento de las instituciones universitarias. En el Ecuador le correspondió al Consejo Nacional de Universidades y Escuelas Politécnicas (CONUEP), asumir el liderazgo para introducir el concepto sobre evaluación en el sistema de educación superior, que ya constituía una exigencia de la sociedad ecuatoriana y de la comunidad académica en particular que demandaban transparencia en la gestión académica, administrativa y financiera de las universidades. (CONEA, 2009).

La evaluación institucional universitaria recobró importancia debido a las exigencias de la sociedad por lograr un sistema educativo de calidad, pasando de ser concebida como un proceso burocrático dirigido por agencias que utilizan modelos de evaluación complacientes, por constituir un proceso necesario e indispensable para dar respuesta a las demandas del estado y de la sociedad.

La evaluación no debe ser entendida como una actividad más de la institución, porque tiene una finalidad suprema que es mejorar la calidad educativa, que siendo un concepto abstracto y relativo, tiene un carácter comparativo con el que debe confrontarse, tanto, desde su propia naturaleza, su proyecto educativo institucional, sus objetivos, etc., lo que en suma permite conocer las debilidades y fortalezas de la institución educativa y proyectar sus acciones al mejoramiento de los servicios de una manera planificada para el cumplimiento de su misión y, a su vez, satisfacer las demandas de la sociedad. (UNESCO, 2005).

En todo proceso de evaluación se utilizan modelos o enfoques que contemplan dimensiones, criterios e indicadores, que han sido aplicados en diferentes organizaciones, de allí que serán parte de análisis en esta investigación: Modelo de evaluación de Tyler, Modelo de evaluación libre de Scriven, Evaluación respondiente de Stake, Modelo CIPP de Sttufflebeam, Modelo de la calidad total EFQM, Modelo CINDA, CEAACES, entre otros; que tienen como objetivo común la búsqueda de la calidad y que son aplicables por tanto en el contexto de las instituciones universitarias, como es el caso de la universidad ecuatoriana en los últimos diez años.

El presente artículo de investigación busca examinar los modelos de evaluación institucional que han sido utilizados en el contexto universitario, lo que permite hacer una aproximación a las coincidencias y divergencias surgidas en la aplicación de dichos modelos.

\section{Referentes conceptuales}

Existen diferentes enfoques y definiciones al referirse a la evaluación. (Stufflebeam, 1987), señala que:

Es el proceso de identificar, obtener y proporcionar información útil y descriptiva acerca del valor y el mérito de las metas, la planificación, la realización y el impacto de un objetivo determinado, con el fin de servir de guía para la toma de decisiones, solucionar los problemas de responsabilidad y promover la comprensión de los fenómenos implicados (p. 183). 
Para el (CONEAU, 1997), la evaluación institucional constituye un instrumento que permite la transformación de las universidades, para cuya práctica es indispensable llevar adelante un proceso constructivo, participativo, consensuado, sistemático y permanente para detectar las debilidades y fortalezas, tanto en los aspectos cualitativos como cuantitativos que incluyen los insumos, procesos, productos y el impacto que tienen que se genera en la sociedad.

(Casanova, 2004), afirma que en la evaluación se desarrollan un conjunto de acciones dirigidas, planificadas, organizadas de una manera integral que requiere la participación de todos los actores de la propia institución, con el objetivo de contribuir en su renovación y transformación permanente, conocer todo lo que se ha realizado y mejorado, para tomar decisiones a partir de la elaboración y ejecución de planes de fortalecimiento.

Según (Pose, 2008), la evaluación institucional, se puede como sinónimo de medición; de juicio profesional; como el proceso de comparar datos con requerimientos específicos; como el proceso de identificar y reunir información para para los organismos que toman decisiones, en tal sentido, la evaluación es el proceso integral para mejorar la calidad educativa.

Todo proceso de evaluación se realiza teniendo de base un modelo que contenga mínimamente estrategias, criterios, indicadores, con algún grado de integración que permita efectuar un análisis de la situación imperante en la institución en los ámbitos de la gestión académica y administrativa, contando con la participación de sus propios integrantes.

Las diferentes organizaciones relacionadas o no con el quehacer educativo, desde su misión pretender contar con procesos y procedimientos que les permita lograr la calidad que se expresa en la satisfacción de los usuarios de los servicios que prestan y en esta medida han utilizado diferentes modelos de evaluación sustentados, muchos de ellos, en sus propias dimensiones, criterios e indicadores, que es necesario develar a través de su análisis para conocer sus debilidades y fortalezas, para con estos elementos contar con fundamentos teóricos de cómo se ha realizado la evaluación institucional, especialmente en el caso ecuatoriano.

Los modelos de evaluación han tenido sus épocas de auge y han evolucionado de acuerdo con los períodos y las reformas educativas, cada uno de ellos con aspectos positivosnegativos y generalmente respondiendo a posturas epistemológicas o al momento que atraviesa la universidad y las decisiones que adoptan los diferentes organismos de control y regulación de la educación superior. (González, 1996).

En este artículo se aborda la evaluación institucional desde la perspectiva teórica de los autores creadores de los modelos, dado que cada uno han sido concebidos en un tiempo y en un espacio histórico que ha generado una serie de interrogantes que fundamentan las dimensiones, indicadores, criterios que lo sustentan.

\section{Metodología}

La investigación realizada no se propuso la comprobación de hipótesis a partir de evidencia empírica que pueda ser interpretada. En su lugar, la investigación se propuso un análisis documental, utilizando la técnica de análisis de contenido que supone estudiar los 
contenidos de un material previamente seleccionado de diferentes modelos de evaluación institucional aplicados en organizaciones y de manera particular el contexto universitario, para establecer algunas semejanzas y divergencias de la evaluación en el entorno de aprendizaje, que permita entender los procesos de evaluación que están orientados a la mejora de la calidad del servicio educativo que ofrecen las instituciones de educación superior.

\section{Resultados}

Para alcanzar el propósito de esta investigación se hace un recorrido por diferentes modelos de evaluación que han sido aplicados, lo que permite hacer un acercamiento a los procesos de evaluación llevados a cabo en función de los postulados que cada uno de ellos considera y los resultados que se pretende alcanzar para lograr el mejoramiento de la calidad educativa.

En el ámbito educativo existen modelos que han puesto énfasis en la evaluación de programas, para la toma de decisiones, la gestión de calidad, la evaluación de competencias, y la evaluación institucional, que se han ido adaptando a los requerimientos de la sociedad que exige de las universidades una educación coherente con las necesidades del país y de los organismos de control el aseguramiento de la calidad.

Los modelos permiten identificar los elementos claves que requieren ser medidos dentro del sistema, por lo que de una manera breve se presentan los elementos centrales de algunos de ellos que por su contenido son parte de este análisis y aquellos que más específicamente han sido aplicación en la evaluación de la universidad ecuatoriana:

1. La evaluación orientada al logro de los objetivos de Tyler: para Tyler, la referencia central en la evaluación son los objetivos preestablecidos, que deben ser cuidadosamente definidos en términos de conducta, teniendo en cuenta que deben marcar el desarrollo individual del alumno. La propuesta de Tyler se orienta a la evaluación de programas, determinando los objetivos propuestos y los logros alcanzados para tomar las decisiones que correspondan en procura del mejoramiento del proceso educativo.

2. La evaluación orientada a la toma de decisiones para la mejora de Stufflebeam: uno de los modelos más prácticos es el modelo que propone este autor, que se lo conoce también como modelo CIPP, (Contexto, insumo, producto y proceso), o toma de decisiones, que constituye un referente para el posterior desarrollo de otros modelos entre ellos sobre la evaluación institucional. (Herreras, 2003).

(Stufflebeam, 1987), afirma que la evaluación es un proceso que aporta información relevante para toma de decisiones, ya que se lo define como un estudio sistemático planificado, que conduce o coadyuva a que los usuarios puedan valorar el mérito que tiene el producto o servicio que se ofrece.

3. Evaluación sin referencia a objetivos de Scriven: para (Scriven, 1967), la evaluación es el proceso sistemático de recogida de datos que permite obtener información válida y confiable de las necesidades de los usuarios sin privilegiar los objetivos que se encuentran predeterminados, las mismas que son definidas de forma externa y objetiva por parte de un evaluador, lo que le permite hacer juicios de valor acerca del desempeño de la institución educativa. 
4. Modelo de evaluación sensitivo o respondiente propuesto por Robert Stake: según (Fonseca, 2007), el análisis de los antecedentes, los procesos y los juicios, de la información obtenida antes de la ejecución de un programa educativo, los procesos que se generan durante la ejecución y los resultados en sus diversos aspectos y componentes, son postulados del modelo de Stake, que buscan responder a las necesidades de los usuarios, tomando en cuenta el contexto, las perspectivas, e intereses particulares y colectivos como actores del sistema que interaccionan y profundizan las relaciones causa - efecto de los entre los resultados esperados y los contingentes.

5. Modelo europeo para la gestión de la calidad total (EFQM): el EFQM contiene factores o criterios que están interrelacionados y es aplicable a cualquier organización, y en esencia propone realizar una evaluación de los diferentes elementos que la integran como: valores, modelo de gestión, procesos, resultados, etc., y compara los resultados con los elementos teóricos que lo definen.

El Modelo EFQM está concebido para hacer una evaluación global, profunda y sistemática que permite identificar y proponer procesos de mejora en cualquier organización para garantizar la calidad de sus productos o servicios sin que esto tenga que ver con el tamaño, el carácter o el sector al que pertenezcan, de allí la amplitud para su aplicación. (Colmenares \& López, 2013).

6. Modelo de evaluación de Gento Palacios: tiene un enfoque agregado o total porque incluye en la evaluación a todos los componentes y las relaciones que se establecen en el proceso educativo para alcanzar resultados positivos en coherencia con lo que la educación deben ser las instituciones educativas, utilizando en su modelo indicadores que de acuerdo a su nivel de cumplimiento permitan evidenciar procesos de calidad, y a su vez, predictores que no son más que características que deben de reunir las instituciones educativas para asegurar que dicha calidad se va a producir a lo interno, beneficiando con ello a los actores externos y a la sociedad.

7. Modelo de evaluación de la calidad de CINDA: el modelo del Consorcio Interuniversitario de Desarrollo (CINDA), contempla seis dimensiones: relevancia, efectividad, disponibilidad de recursos, eficiencia, eficacia, y procesos. A su vez, las dimensiones consideran criterios para garantizar el cumplimiento de las mismas, y estos criterios, a su vez, contemplan indicadores y variables cuantitativas y cualitativas para asegurar la calidad en las funciones de docencia, investigación, extensión y gestión de las instituciones universitarias en base a la data que proporcionan los actores de la comunidad universitaria, esto es, alumnos, profesores y personal administrativo lo que permite determinar las fortalezas y debilidades de la institución, lo que permite establecer estrategias de desarrollo institucional. (CINDA, 1994).

8. Los modelos de evaluación CONEA - CEAACES - CACES: en el Ecuador, el Consejo Nacional de Evaluación y Acreditación (CONEA), en el año 2009 elabora el primer modelo de evaluación de desempeño institucional de las instituciones de educación superior, cuyo propósito es buscar la calidad de la educación superior, considerando tres etapas básicas: planeación, realización y presentación de resultados.

Para el (CONEA, 2009), el modelo de evaluación institucional se lo define como: integral y participativo que pretende mejorar e innovar la educación superior del país, 
reconociendo las particularidades de cada institución pero que permite, a su vez, evaluar la calidad entendida como la capacidad para realizar y alcanzar sus fines declarados en su propia filosofía institucional. El modelo considera criterios, subcriterios e indicadores conforme a lo que se observa en la Tabla 1.

Tabla 1

Criterios, Subcriterios e Indicadores M14

\begin{tabular}{lcc}
\hline \multicolumn{1}{c}{ CRITERIO } & SUBCRITERIO & INDICADORES \\
\hline 1. Academia & 11 & 19 \\
2. Estudiantes & 9 & 16 \\
3. Investigación & 5 & 9 \\
4. Gestión & 5 & 9 \\
TOTALES: & 20 & 53 \\
\hline
\end{tabular}

Fuente: El autor

Una vez que se ejecuta el proceso de evaluación institucional, el informe presentado por el CONEA a finales de 2009, provocó una gran alarma en la sociedad ecuatoriana, debido a que los resultados evidenciaron las preocupaciones que existían sobre el manejo que se venía dando en el sistema de educación superior, y de manera especial en las universidades particulares que funcionaban sin reunir las condiciones mínimas que debe cumplir una institución universitaria desde lo legal, académico, infraestructura, organización etc.

En el informe en mención, de acuerdo a su desempeño las universidades fueron clasificadas en cinco categorías: A, B, C, D y E, siendo la A, la de mejor desempeño y la E la de más bajo desempeño. (CONEA, 2009).

Sobre la base normativa vigente (LOES 2010), el Consejo de Evaluación, Acreditación y Aseguramiento de la Calidad de la Educación Superior (CEAACES), define un nuevo modelo de evaluación institucional que toma en cuenta el principio de calidad contemplado en la ley y las particularidades del sistema de educación superior ecuatoriano. (CEAACES, 2012).

El modelo consideró cinco criterios para el entorno de aprendizaje: academia, eficiencia académica, investigación, organización e infraestructura, con subcriterios e indicadores; y los criterios de competencias generales y específicas en el componente resultados de aprendizaje, como se observa en la Tabla 2.

Tabla 2

Modelo de Evaluación Institucional 2012

\begin{tabular}{lcccc}
\hline \multirow{2}{*}{ COMPONENTES } & CRITERIO & SUBCRITERIO & SUBCRITERIO & INDICADORES \\
& & $\mathbf{1}$ & $\mathbf{2}$ & \\
\hline $\begin{array}{l}\text { 1.- Entorno de } \\
\text { aprendizaje }\end{array}$ & Academia & 2 & 6 & 17 \\
& Currículo e & 2 & 3 & 11 \\
& Investigación & & 4 & 13 \\
& Infraestructura & 3 & & 6 \\
\hline
\end{tabular}

Esta obra se comparte bajo la licencia Creative Common Atribución-No Comercial 4.0 International (CC BY-NC 4.0) Revista de la Universidad Internacional del Ecuador. URL: https://www.uide.edu.ec/ 


\begin{tabular}{|c|c|c|c|c|}
\hline COMPONENTES & CRITERIO & $\begin{array}{c}\text { SUBCRITERIO } \\
1\end{array}$ & $\begin{array}{c}\text { SUBCRITERIO } \\
2\end{array}$ & INDICADORES \\
\hline Subtotales: & & 9 & 13 & 47 \\
\hline \multirow{3}{*}{$\begin{array}{l}\text { 2.- Resultados de } \\
\text { aprendizaje }\end{array}$} & \multirow{2}{*}{$\begin{array}{l}\text { Competencias } \\
\text { Generales }\end{array}$} & 1 & & 1 \\
\hline & & 1 & & 1 \\
\hline & $\begin{array}{c}\text { Competencias } \\
\text { Específicas }\end{array}$ & 1 & & 1 \\
\hline Subtotales: & & 3 & & 3 \\
\hline TOTAL: & & 12 & 13 & 50 \\
\hline
\end{tabular}

Fuente: El Autor

Como resultado de la evaluación se definieron cuatro categorías, distribuidas de la siguiente manera: categoría A (5 universidades); categoría B (23 universidades); categoría C (18 universidades); categoría D (8 universidades). Tres universidades no fueron evaluadas y su acreditación quedó pendiente. Las universidades de categorías A, B y C fueron acreditadas, y las de categoría D quedaron en proceso de acreditación. (CEAACES, 2013).

En el año 2013 el CEAACES inicia un nuevo proceso de evaluación a las universidades y escuelas politécnicas ubicadas en categoría "D", para este proceso se define un modelo en el que se incluyen criterios, subcriterios e indicadores, conforme a lo que se observa en la Tabla 3 que a continuación se detalla:

Tabla 3

Modelo de Evaluación 2013

Criterios, Subcriterios e Indicadores

\begin{tabular}{lcc}
\hline \multicolumn{1}{c}{ CRITERIOS } & SUBCRITERIOS & INDICADORES \\
\hline A. ACADEMIA & 9 & 16 \\
B. EFICIENCIA ACADÉMICA & 4 & 6 \\
C. INVESTIGACIÓN & - & 4 \\
D. ORGANIZACIÓN & - & 9 \\
E. INFRAESTRUCTURA & 3 & 8 \\
TOTAL: 5 & 16 & 46 \\
\hline
\end{tabular}

Fuente: El Autor

En el informe presentado por el CEAACES, se determina que las universidades que dejaron la categoría D, siendo re categorizadas la Universidad Católica de Cuenca, en la categoría B; las otras tres universidades fueron ubicadas en la C, siendo estas la Universidad Laica Eloy Alfaro, de Manabí; Luis Vargas Torres, de Esmeraldas, y Agraria del Ecuador. (CEAACES, 2016).

En el marco del proceso de evaluación institucional a universidades y escuelas politécnicas se elaboró un nuevo modelo de evaluación tomando como base el modelo 2013 (CEAACES, 2015), para proceso de re categorización solicitado por varias universidades, conforme a los siguientes criterios, subcriterios e indicadores que están relacionados con las funciones sustantivas de la universidad, así como a las condiciones de apoyo necesarias para su funcionamiento, como se explican en la Tabla 4.

Esta obra se comparte bajo la licencia Creative Common Atribución-No Comercial 4.0 International (CC BY-NC 4.0)

Revista de la Universidad Internacional del Ecuador. URL: https://www.uide.edu.ec/ 


\section{Tabla 4}

Modelo de Evaluación Institucional 2015

\begin{tabular}{lcc}
\hline \multicolumn{1}{c}{ CRITERIOS } & $\begin{array}{c}\mathrm{N}^{\circ} \\
\text { SUBCRITERIOS }\end{array}$ & $\begin{array}{c}\mathrm{N}^{\circ} \mathrm{DE} \\
\text { INDICADORES }\end{array}$ \\
\hline 1. Organización & 3 & 8 \\
2. Academia & 3 & 13 \\
3. Investigación & 2 & 5 \\
4. Vinculación con la Sociedad & 2 & 3 \\
5. Recursos e Infraestructura & 3 & 9 \\
6. Estudiantes & 2 & 6 \\
TOTAL & 15 & 44 \\
\hline
\end{tabular}

Fuente: El Autor

Del informe presentado por el CEAACES, seis universidades lograron aprobar el proceso de evaluación y fueron recategorizadas, tres de ellas fueron ubicadas en la categoría A, y tres a la categoría B. En los resultados del informe se justificó que seis universidades instituciones permanezcan en la categoría $\mathrm{C}$ por no haber alcanzado los niveles de cumplimiento exigidos en el modelo para subir de categoría. (CEAACES, 2016).

En el año 2018 se reforma la Ley Orgánica de Educación Superior, y le corresponde al nuevo Consejo de Aseguramiento de la Calidad de la Educación Superior (CACES), diseñar y construir el modelo de evaluación institucional al amparo de la normativa vigente, cuya premisa principal se basa en garantizar la calidad más que la acreditación universitaria. El modelo del CACES que ha sido socializado y aprobado para el proceso de evaluación que por mandato legal debe realizarse en el año 2019, considera como pilares sujetos a evaluación las tres funciones sustantivas de la universidad ecuatoriana: docencia, investigación e innovación, vinculación con la sociedad. (CACES, 2019).

A estos tres ejes o núcleos del modelo de evaluación se añade otro, de carácter general y transversal a las funciones sustantivas, denominado en el modelo "Condiciones institucionales", que se refiere a aspectos tangibles e intangibles indispensables para su funcionamiento y que por tanto constituyen los pilares sobre los que se levanta el ejercicio de las funciones sustantivas como se observa en la Tabla 5.

\section{Tabla 5}

Ejes, dimensiones y número de estándares del modelo de evaluación institucional 2019

\begin{tabular}{|c|c|c|c|c|}
\hline \multirow[t]{2}{*}{ Ejes de la evaluación } & \multicolumn{3}{|c|}{ Dimensiones de la evaluación } & \multirow{2}{*}{$\begin{array}{l}\text { Total de } \\
\text { estándares }\end{array}$} \\
\hline & Planificación & Ejecución & Resultados & \\
\hline $\begin{array}{l}\text { Función sustantiva Docencia } \\
\text { (Profesorado y estudiantado) }\end{array}$ & 2 & 2 & 4 & 7 \\
\hline $\begin{array}{l}\text { Función sustantiva } \\
\text { Investigación }\end{array}$ & 1 & 1 & 3 & 5 \\
\hline $\begin{array}{l}\text { Función sustantiva Vinculación } \\
\text { con la Sociedad }\end{array}$ & 1 & 11 & & 3 \\
\hline Condiciones institucionales & & & TOTAL & $\begin{array}{c}7 \\
22\end{array}$ \\
\hline
\end{tabular}

Fuente: El Autor

El objetivo principal de los procesos de evaluación impulsados por el CONEA, el Consejo de Evaluación, Acreditación y Aseguramiento de la Calidad de la Educación 
Superior (CEAACES), ha sido el mejoramiento de la educación superior y la calidad en sus procesos, y en esa ruta se han definido y aplicado diferentes modelos de evaluación los cuales con algunas variantes han enfocado la evaluación a las dimensiones de: Academia, Eficiencia Académica, Investigación, Vinculación con la Sociedad, Organización e Infraestructura.

Lo que puede observarse en las evaluaciones realizadas por el CONEA - CEAACES, es que los cambios realizados, al menos a nivel de criterios del modelo de evaluación, no fueron sustantivos. Los elementos que sustentan las dimensiones y criterios como los subcriterios e indicadores de la evaluación es donde más cambios se han realizado, con la pretensión de lograr un equilibrio entre los elementos cualitativos y cuantitativos. Las diferentes modificaciones en los criterios del modelo, a lo largo de las sucesivas evaluaciones, coloca en entredicho los diagnósticos realizados en su momento tanto por el CONEA como por el CEAACES, al definir y aplicar modelos cuyos objetivos y dimensiones no eran lo suficientemente claros, sin contar con una mayor participación de los actores del sistema y que generaron resultados ambiguos y no permitieron asegurar la calidad de la educación superior y más bien se constituyeron en una lista de cumplimiento para las universidades a las que debían dedicarles toda la atención para superar los procesos de evaluación institucional.

A pesar de presentar una estructura diferente, el modelo de evaluación del CACES considera la evaluación como un proceso basado en las dimensiones de planificación, ejecución y resultados, que articula a las tres funciones sustantivas de la universidad, contando con los apoyos que deben garantizarse mínimamente en lo que se ha denominado "condiciones institucionales", cuyos estándares son muy similares a los que estaban presentes en los anteriores modelos de evaluación de los organismos que precedieron al CACES y en otros como el caso de CINDA, Gento Palacios y FQM, cuyas características básicas constan en este análisis, y su aplicación y resultados dependerá en alto grado del criterio de los pares evaluadores más aún cuando de los 22 estándares, más del cincuenta por ciento son cualitativos y las escalas de valoración tienen amplios niveles de amplitud que podrían generar resultados con altos índices de subjetividad en el proceso de acreditación de las universidades ecuatorianas.

\section{Conclusiones}

Todos los modelos de evaluación que se exponen que han sido utilizados en diferentes ámbitos y organizaciones que buscan alcanzar la calidad del servicio o de la empresa según sea el caso.

En todos los modelos existen elementos que aportan para realizar una evaluación curricular, de programas o la evaluación institucional.

Cada uno de los autores de los diferentes modelos tienen definido metas y el procedimiento para su aplicación, siendo algunos como los de Tyler y Scriven, más enfocados a los objetivos, y otros como los de Sttufflebeam y Stake que toman en cuenta para la evaluación variables cuantitativas y cualitativas, en este último caso se pueden ubicar aquellos que se diseñaron y ejecutaron en los procesos de evaluación de la universidad ecuatoriana. 
Los autores de los modelos que han sido referidos en este artículo, con sus propuestas han contribuido significativamente a la evaluación y sobre todo a que los resultados sean utilizados para mejorar la educación en sus diferentes niveles.

Los modelos EFQM y de Gento Palacios, coinciden en la búsqueda de la calidad educativa y de la calidad total de las organizaciones.

Los modelos CINDA y CONEA - CEAACES, están identificados directamente por sus dimensiones y criterios con la evaluación institucional de las universidades que buscan la calidad educativa, la acreditación de las IES y su fortalecimiento, y en el Ecuador han sido últimos aplicados con resultados favorables y otros que han merecido críticas de la comunidad universitaria por los elementos que los contienen, los mecanismos utilizados, los pesos de los criterios, entre otros aspectos.

Existen diferencias en los modelos de los diferentes autores, las mismas que radican en los objetivos que persiguen, la metodología, el ámbito de aplicación, y los destinatarios a los que está dirigido el proceso de evaluación. No todos los modelos en su estructura identifican dimensiones, sólo establecen el qué y para qué de la evaluación y los criterios a evaluar, especialmente cuando se trata de autores como el caso de Tayler.

Los modelos EFQM, CINDA, CONEA - CEAACES - CACES, si bien recogen los postulados de los principales autores de la evaluación de programas, tienen como propósito la calidad de las instituciones, y sus modelos proponen una evaluación que garantice un servicio responda a las necesidades del usuario, pero que si bien su objetivo está orientado a la búsqueda y aseguramiento de la calidad, no necesariamente responden a una realidad concreta de las universidades y es justamente ésta una debilidad de los modelos en logro de la calidad, lo que ha derivado en que se conviertan en instrumentos de cumplimiento de requisitos para alcanzar la acreditación.

Cada modelo de evaluación tiene una determinada forma de concebir la enseñanza, la metodología y los resultados que pueden obtenerse, y pueden ser viables si se determina con claridad el objetivo o la finalidad que se tenga al momento de la evaluación, de allí que sus posibilidades de alcanzar resultados adecuados dependen del contexto y del propósito que se desea alcanzar. De allí que es importante que los organismos responsables de llevar adelante los procesos de evaluación determinen con precisión lo que se quiere evaluar y el propósito que se quiere alcanzar, lo que necesariamente debe ir acompañado de una amplia participación de los actores involucrados en el proceso, para definir un modelo de evaluación que tome en cuenta la realidad y el entorno para realmente ir hacia un aseguramiento de la calidad, que en el caso del Ecuador ya constituye una norma legal de obligatorio cumplimiento para obtener la acreditación universitaria.

Finalmente, en los en los procesos de evaluación es necesario que se genere un ambiente de confianza hacia las instituciones universitarias a ser evaluadas, se propicie la participación, las dimensiones del modelo, los criterios, subcriterios e indicadores y no se limite a ser un proceso eminentemente controlador, sino, de acompañamiento desde el estado hacia la universidad, porque no se trata de eliminar la evaluación sino de que ésta contribuya al mejoramiento de los procesos de enseñanza y demás funciones sustantivas de la universidad para que aporte desde su ámbito al desarrollo del país. 


\section{Bibliografía}

CACES. (2019). Modelo de Evaluación Institucional de Universidades y Escuelas Politécnicas. Quito.

Casanova, M. A. (2004). Evaluación y calidad de centros educativos. Madrid España. Revista Interuniversitaria de Formación del Profesorado.

CEAACES. (2012). Modelo de Evaluación Institucional de de Universidades y Escuelas Politécnicas. Quito.

CEAACES. (2013). Informe General sobre la Evaluación y Acreditación y categorización de las Universidades y Escuelas Polit \{ecnicas. Quito.

CEAACES. (2015). Modelo de Evaluación Institucional de Universidades y Escuelas Politécnicas. Quito.

CEAACES. (2016). Informe Final de Evaluación y Recategorización de Universidades y Escuelas Politécnicas. Quito.

CEAACES. (2016). Proceso de Recategorización 2015. Informe Final. Quito.

CINDA. (1994). Manual de autoevaluación para instituciones de educación superior. Pautas y procedimientos. CINDA/PROMESUP - OEA.

Colmenares, A. R., \& López, D. V. (2013). Introducción a los sistemas de gestión de la calidad total: Modelo de excelencia EFQM y Autoevaluación. Medicina y Seguridad del Trabajo.

CONEA. (2009). Modelo de evaluación de desempeño institucional de las instituciones de educación superior. Quito.

CONEAU. (1997). Lineamientos para la evaluación institucional. Buenos Aires. Argentina: Comisión Nacional de Evaluación y Acreditación Universitaria. .

Fonseca, J. G. (2007). Modelos cualitativos de evaluación. EDUCERE. Revista Venezolana de Educación, 427-432.

González, I. (1996). Modelos de evaluación de calidad orientados a la mejora de las instituciones educativas. Revista de Educación. Universidad de Huelva. España.

Herreras, E. B. (2003). Metodología de la Investigación Educativa: Modelo CIPP. Revista Complutense de Educación, 361-376.

Pose, G. (2008). El estrés en la evaluación institucional. Recuperado de: . Obtenido de http://evaluacioninstitucional.idoneos.com/

Scriven, M. (1967). Perspectives of curriculum evaluation. Chicago: Asociación Americana de Investigación Educativa (AERA).

Stufflebeam, D. (1987). Evaluación Sistemática. Guía teórica y práctica. Editorial PAIDOS, Bercelona, España.

UNESCO. (2005). Informe mundial de la UNESCO. Hacia las sociedades del conocimiento. Paris: Organización de las Naciones Unidas para la Educación, la Ciencia y la Cultura. 